\title{
Development and Validation of a HPLC-MS/MS Assay for the Determination of Lutein Concentration in Human and Rat Plasma
}

\author{
Feng $\mathrm{Wu}^{1}$, Xiuli Zhao ${ }^{1 *}$, Shumin Wang ${ }^{1}$, Hui $\mathrm{Zhou}^{1}$, Shaojie Guo ${ }^{1}$, Siyang Ni ${ }^{1}$, Bo Yang ${ }^{1}$, \\ Lihua Zhang ${ }^{2}$ and Xinde $\mathrm{Xu}^{2}$ \\ ${ }^{1}$ National Institute for Drug Clinical Trial, Beijing Tongren Hospital, Capital Medical University, Beijing-100730, China \\ ${ }^{2}$ Zhejiang Medicine Co. Ltd, Xinchang Pharma. Factory, Zhejiang Xinchang-312500, China
}

Received: 24 April 2017; accepted: 01 October 2017

\begin{abstract}
The aim of this study was to develop and validate a HPLC-MS/MS assay to determine the lutein concentration in plasma samples of human and SD rats. Organic solvent was used for lutein extraction. The extract was injected into a HPLC-MS/MS system. Reversed phase chromatography was performed on a C18 column in gradient mode. Lutein and internal standard (phenytoin sodium) were identified in atmospheric pressure chemical ionization mode using ion transitions of $\mathrm{m} / \mathrm{z} 567.5>549.4$ and 205.2 $>110.8$, respectively. The lutein quantification assay was linear over concentrations ranging from 4 to $500 \mathrm{ng} / \mathrm{mL}$. The lower limit of quantification was $4 \mathrm{ng} / \mathrm{mL}$ with satisfactory precision and accuracy. The assay presented acceptable intra and inter-batch precision $(\mathrm{RSD} \%)$ and accuracy $(\mathrm{RE} \%)<8.16 \%$ in $\mathrm{SD}$ rat plasma and $<12.80 \%$ in human plasma. The extraction recovery ranged from 50.94 to $60.90 \%$ in SD rat plasma and $68.73 \%$ in human plasma. The matrix effect for lutein was acceptable and had minimal influence on the results. The method was then applied to determine the lutein concentrations in human plasma after a single oral dose of $20 \mathrm{mg}$ lutein. The method described is rapid, selective, sensitive and reproducible. This method can be used for both pharmacokinetic studies and therapeutic drug monitoring purposes.
\end{abstract}

Keywords: Lutein, HPLC-MS/MS, Plasma concentration, Pharmacokinetics

\section{Introduction}

Lutein is a xanthophyllic carotenoid found in fruits and vegetables, and has been described as a natural antioxidant. Humans are not capable of synthesizing carotenoids, and thus, their presence in human tissues is entirely of dietary origin. Adequate supply of the dietary carotenoids including lutein and zeaxanthin depends on regular intake of fruits and vegetables. The combined daily dietary intake of lutein plus zeaxanthin ranges, on average, between 2 and $26 \mathrm{mg}$ [1].

Lutein is found in a number of human tissues but the highest concentration of these carotenoids $(0.1-1 \mathrm{mM})$ is found in the human retina [2]. In the human eye, lutein and zeaxanthin are specifically located at the center of the retina. Evidence indicates that poor dietary intake or low plasma concentrations of lutein and zeaxanthin are associated with low macular pigment optical density (MPOD) and an increased risk potential for age-related macular degeneration (AMD), an irreversible ocular condition that is the major cause of blindness in the elderly [3]. In several randomized placebo-controlled trials among patients with early AMD, supplementation with lutein alone or in conjunction with zeaxanthin or docosahexaenoic acid (DHA) [4] increased MPOD significantly during 48-week [5], 12-month [6], and 2-year periods [7]. This played a beneficial effect on visual acuity and might prevent the progression of AMD.

One of the most rigorous studies showed that supplementation with 4.1 and $20.5 \mathrm{mg}$ lutein increased plasma lutein concentrations approximately 3.5 and 10 fold, respectively [8]. On the basis of previous studies, it may be hypothesized that dietary lutein and zeaxanthin in the form of lutein-containing supplements may increase the amount of serum lutein significantly [9]. In a clinical trial where two formulations of lutein and zeaxanthin

*Author for correspondence: lilyzhao1028@aliyun.com supplements were administered to Japanese subjects, the mean serum lutein concentrations increased significantly after the first three months [10].

Two validated assays have been suggested to quantify lutein in human plasma and have been used for pharmacokinetic study of lutein supplements. The first was used for comparing the bioavailability of two different lutein formulations and confirmed superiority of the starch-based product over the alginate-based one [11]. The other [12] was used for characterizing lutein plasma kinetics in a multiple dosing design. Both methods involve an ultraviolet-visible (UV) detector. Besides, in another study investigating the pharmacokinetics of lutein emulsion following oral administration to SD rats, the plasma concentration of lutein was also determined by a HPLC-UV assay [13]. The methods were then improved for the determination of possible lutein oxidation products in plasma and tissues of adult rats. Total ionic chromatogram together with UV-visible and mass spectra were used to differentiate lutein isomers and its oxidative products [14].

The present work aims to validate a new, rapid, sensitive and selective LC-MS/MS (liquid chromatography-tandem mass spectrometry) method for the determination of lutein concentrations in human and SD rat plasma. Because of the obvious background concentration of lutein in human, the drug-free SD rat plasma was used to prepare the calibrator in this assay. In order to ensure feasibility, the method was validated in both human and SD rat plasma.

\section{Experimental}

Chemicals and Reagents. Lutein (purity: 97\%, Lot: 140414) was obtained from Zhejiang Medicine Co. Ltd (ZMC), Xinchang Pharma. Factory (Xinchang, China).

This is an open-access article distributed under the terms of the Creative Commons Attribution-NonCommercial 4.0 International License (https://creativecommons.org/licenses/by-nc/4.0/), which permits unrestricted use, distribution, and reproduction in any medium for non-commercial purposes, provided the original author and source are credited, a link to the CC License is provided, and changes - if any - are indicated. 
Phenytoin sodium (purity: 99\%, Lot: 100210-201303; internal standard, IS) was procured from National Institutes for Food and Drug Control (Beijing, China). HPLC/MS grade methanol, acetonitrile and isopropyl alcohol were purchased from HoneyWell (Wicklow, IE, USA). HPLC/MS grade Acetic acid was obtained from Dikma (Ohio, USA). Dimethyl sulfoxide (DMSO) and 2,6-Di-tert-butyl-p-cresol (BHT) were from Sinopharm Chemical Reagent Co. Ltd (Beijing, China). Methyl-tert-Butyl Ether was purchased from Fisher Scientific (Pittsburgh, PA, USA). Ultrapure water $(\geq 18.3 \mathrm{M} \Omega)$ was obtained from Nanopure water purification system of Thermo scientific (Marietta, OH, USA). Human drug-free plasma was obtained from healthy volunteers, in accordance with the local ethics guidelines.

Instrumentation. The HPLC-MS/MS equipment consisted of an API-4000 mass spectrometry from AB company (Framingham, MA, USA) fitted with a Turbo Ionspray ion source. The instrument was operated in Atmospheric Pressure Chemical Ionization $\left(\mathrm{APCI}^{-}\right)$coupled to an Agilent 1100 Series HPLC system from Agilent Technologies (CA, USA) with an integrated auto sampler (LEAP Technologies, Basle, Switzerland). Data were recorded in the multiple reaction monitoring (MRM) mode.

Sample Preparation. For the determination of lutein concentrations, $20 \mu \mathrm{L}$ of standard working solution were added into drug-free plasma to prepare 7 calibrators (CS) in the 4 to $500 \mathrm{ng} / \mathrm{mL}$ range. The quality control (QC) samples $(12,100$, and $350 \mathrm{ng} / \mathrm{mL}$ ) were prepared from an independent stock solution. Subsequently, aliquot of each CS or QC was treated with $1000 \mu \mathrm{L}$ of organic solvent (Methyl-tert-Butyl Ether, MTBE) following the addition of $20 \mu \mathrm{L}$ of IS working solution (50 ng/mL). After a 3 minute vortex step samples were centrifuged at $12,000 \mathrm{rpm}$ for $5 \mathrm{~min}$. The organic layer $(0.850 \mathrm{~mL})$ was collected and evaporated under a stream of nitrogen, and the residue was reconstituted in $100 \mu \mathrm{L}$ methanolwater $\left(9: 1, \mathrm{v} / \mathrm{v}\right.$, containing $1.0 \mathrm{mg} \cdot \mathrm{mL}^{-1}$ BHT). This was followed by vortex for about $3 \mathrm{~min}, 10 \mu \mathrm{L}$ water addition and a further vortex for about $1 \mathrm{~min} .25 \mu \mathrm{L}$ of the sample was then injected into the LC-MS/MS system.

Chromatographic Conditions. The analytical column was a Grace Alltima HP C18 $(50 * 2.1 \mathrm{~mm}, 5 \mu \mathrm{m})$ (Berkshire, UK) maintained at $25^{\circ} \mathrm{C}$. The mobile phase consisted of a mixture of solvents A (methanol-water-acetic acid, 10:90:0.06, v/v/v) and B (methanol-acetic acid, 100:0.06, v/v), under gradient conditions and $0.3 \mathrm{~mL} / \mathrm{min}$ flow rate. The auto sampler used a rinse solution consisting of solution A (Acetonitrile-waterformic acid, 10:90:0.1, v/v/v) and B (Methanol-AcetonitrileIsopropyl-alcohol-water-formic acid, 25:25:25:25:0.1, v/v/v/v/v). The total run time was $5 \mathrm{~min}$. The transitions $\mathrm{m} / z \quad 567.5>549.4$ and $m / z$ 205.2>110.8 were used for lutein and IS quantification, respectively. The collision energies (CE) optimized for the method were -29 and $-28 \mathrm{eV}$, respectively for lutein and IS.

Method Validation. The assay was fully validated according to the U.S. Food and Drug Administration (FDA) guidelines [15]. Statistical analysis was performed using Analysis 1.4.2 software.

Specificity was tested by analyzing plasma samples of SD rats from six different sources. The linearity had been assessed over the 7 calibrators in 9 replicates over 3 batches. The lower limit of quantification (LLOQ) was calculated as the minimum concentration at which lutein can be reliably quantified with a precision of $\leq 20 \%$ and accuracy within $\pm 20 \%$.

The intra-batch precision and accuracy were analyzed in 6 replicates in a single batch of SD rat plasma with concentrations at QC level of 12, 100 and $350 \mathrm{ng} / \mathrm{mL}$ and in human plasma with concentrations at $350 \mathrm{ng} / \mathrm{mL}$. Inter-batch precision and accuracy were assessed on the same QC samples in 6 replicates for 5 batches.

Extraction efficiency of lutein from plasma was determined by comparing the peak area ratio of plasma samples treated as described above with unextracted calibrators (6 replicates for the 3 QC concentrations). Matrix effect was assessed by comparing the peak area ratio of unextracted blank plasma samples with neat standard solution prepared at the same QC levels. Extraction recovery and matrix effect for IS were assessed using the same method.

Reinjection stability of extracted samples placed at automatic sampler $\left(4^{\circ} \mathrm{C}\right)$, long-term stability of unextracted samples at $-20^{\circ} \mathrm{C}$, freeze/thaw stability, and bench-top stability of unextracted samples placed at room temperature were processed with QC samples both in SD rat and human plasma.

\section{Results and Discussion}

Method Validation. Lutein maintained its linearity from 4 to $500 \mathrm{ng} / \mathrm{mL}$ when using a $1 / X^{2}$ weighted linear regression. The correlation coefficient $\left(R^{2}\right)$ for each calibration curve was $>0.99$, with a mean value of $0.9994(\mathrm{n}=9)$. The LLOQ was found to be $4 \mathrm{ng} / \mathrm{mL}$. For LLOQ, the precision (RSD\%) was $13.60 \%$ while the accuracy (RE\%) was $1.47 \%$. As shown in Figure 1, the retention times (RT) for lutein and IS were 3.24 and $2.15 \mathrm{~min}$, respectively.

No interfering peak greater than $20 \%$ of the response at the LLOQ was detected at the $\mathrm{m} / \mathrm{z}$ transitions and RT of lutein and IS. Typical ion chromatograms are shown in Figure 1 for extracts from double blank sample (Figure 1A), blank sample spiked with IS (Figure 1B), plasma spiked with IS and lutein at $4 \mathrm{ng} / \mathrm{mL}$ (Figure 1C) and plasma sample from a healthy subject at 3 hours after oral administration of $20 \mathrm{mg}$ lutein (Figure 1D).

For lutein in SD rat plasma, the intra-batch precision (RSD\%) and accuracy (RE\%) ranged from $4.91 \%$ to $7.05 \%$ and from $-0.56 \%$ to $6.43 \%$, respectively. The inter-batch ( 5 batches; $\mathrm{n}=30$ ) precision and accuracy ranged from 6.79 to $8.16 \%$ and from $-4.1 \%$ to $-2.13 \%$, respectively. For lutein in human plasma, the intra-batch precision and accuracy were $2.36 \%$ and $-12.80 \%$, while the inter-batch precision and accuracy were $8.48 \%$ and $-5.35 \%$, respectively.

At the three QC levels, the extraction recovery of lutein ranged from 50.94 to $60.90 \%$ in SD rat plasma and $68.73 \%$ in human plasma. For IS it was $77.23 \%$ in SD rat plasma and $84.08 \%$ in human plasma.

The matrix effect was acceptable, ranging from 94.15 to $109.66 \%$ for lutein in SD rat plasma and $87.59 \%$ in human plasma at the QC levels. For IS it was $105.90 \%$ in SD rat plasma and $88.52 \%$ in human plasma. The results suggested that matrix effects had minimal influence on the results.

Reinjection stability for up to 21 hours at $4^{\circ} \mathrm{C}$, long-term stability for up to 31 days at $-20^{\circ} \mathrm{C}$, freeze/thaw stability for two cycles, and bench-top stability for up to 4.7 hours at room temperature were satisfactory, which covered the expected conditions of processing clinical samples.

Method Development. The HPLC-MS/MS method described in this work for the quantification of lutein in SD rat and human plasma proved to be rapid, sensitive, selective and reproducible. There are several reports concerning the human plasma concentration of lutein, where lutein had been measured by a common method using a UV-visible detector. As MS/MS detection is highly specific, the assay developed in the present work probably has wider applicability to different clinical situations than the previous methods.

The level of lutein concentration in human plasma $(>100 \mu \mathrm{g} / \mathrm{L})$ is substantially higher than the LLOQ $(4 \mu \mathrm{g} / \mathrm{L})$. 


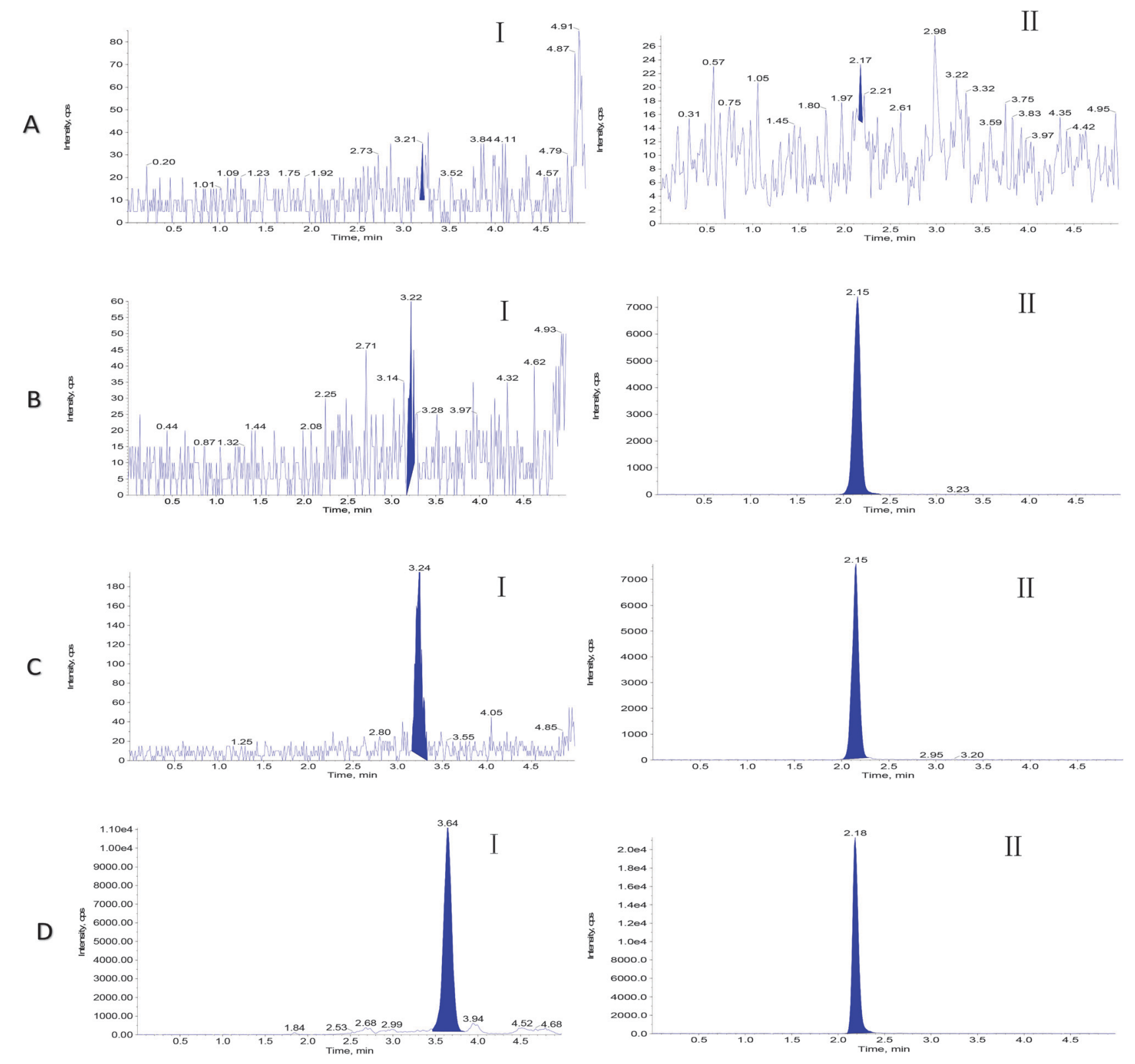

Figure 1. Extracted ion chromatograms of lutein and IS (internal standard) in plasma by LC-MS/MS. (A: double blank SD rat plasma, B: blank SD plasma spiked with IS, C: plasma spiked with IS and lutein at $4 \mathrm{ng} / \mathrm{mL}$, D: plasma sample from a healthy volunteer at 3 hours after oral administration of 20 mg lutein; I: Lutein; II: IS)

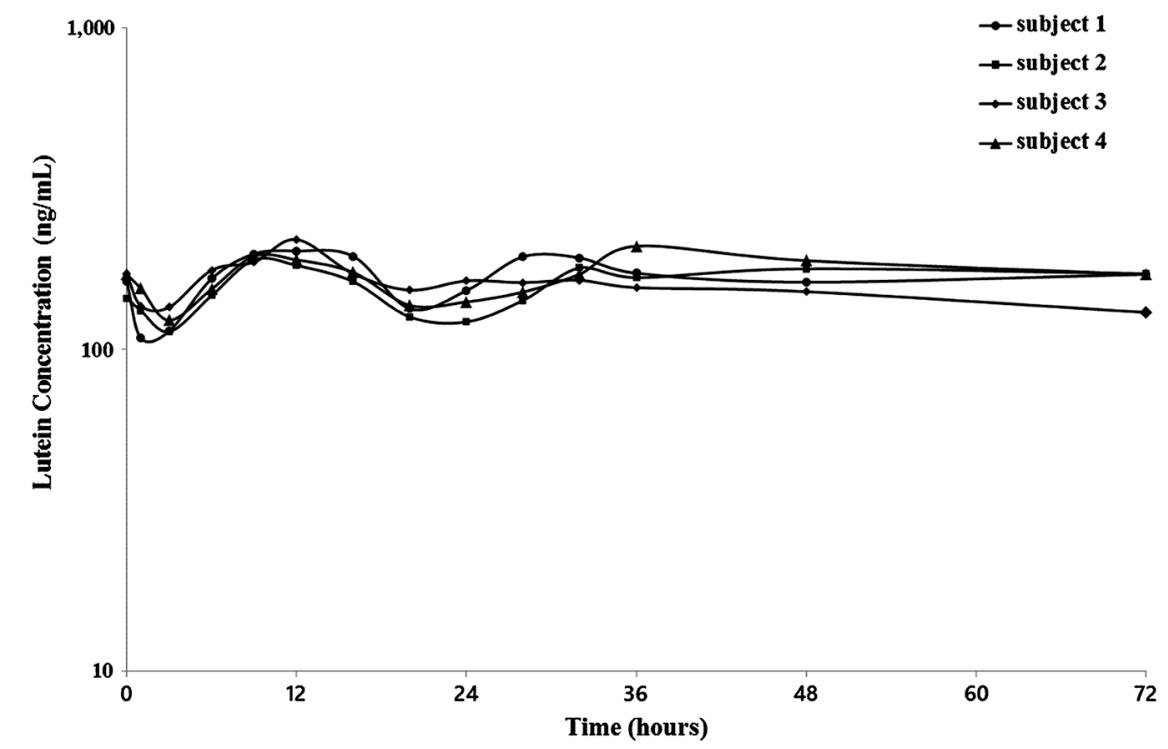

Figure 2. Plasma concentration-time profiles of lutein in 4 individual healthy volunteers following administering a single oral dose of $20 \mathrm{mg}$ lutein. 
Accordingly, the sensitivity of the measurement can fulfill the determination purpose, although it had not been improved in this LC-MS/MS method. However, the new assay, with RT of about $3.4 \mathrm{~min}$, is faster than the common one. Besides, less plasma sample $(20 \mu \mathrm{L})$ is required compared to $100 \mu \mathrm{L}$ used in the previous method [12]. For zeaxanthin, the structural isomers of lutein, the RT was about 3.9 min (Figure 1D), so that it was well separated.

Pharmacokinetic Application. The method was then applied to determine the concentration-time profile of lutein in the plasma of healthy volunteers, after receiving a single oral dose of $20 \mathrm{mg}$ lutein. The four individual timeconcentration curves are shown in Figure 2.

As evidenced from the results, lutein concentration-time profile matched those determined by previously validated HPLC-UV methods [11]. This further confirmed the feasibility and accuracy of the newly developed method during its clinical application. This LC-MS/MS assay will continue to be utilized for drug monitoring in patients receiving lutein supplements, as well as in the pharmacokinetics study of various lutein formulations.

Acknowledgements. This work was partly supported by the Chinese National Major Scientific and Technological Special Project for Significant New Drug Development (2011ZX09302-007-05).

\section{References}

1. Johnson, E. J.; Maras, J. E.; Rasmussen, H. M.; Tucker, K. L. J. Am. Diet. Assoc. 2010, 110, 1357-1362.

2. Landrum, J. T.; Bone, R. A.; Moore, L. L.; Gomez, C. M. Methods Enzymol. 1999, 299, 457-467.

3. Chiu, C. J.; Taylor, A. Exp. Eye Res. 2007, 84, 229-245.

4. García-Layana, A.; Recalde, S.; Alamán, A. S.; Robredo, P. F. Nutrients 2013, 5, 543-551.

5. Ma, L.; Yan, S. F.; Huang, Y. M.; Lu, X. R.; Qian, F.; Pang, H. L.; Xu, X. R.; Zou, Z. Y.; Dong, P. C.; Xiao, X.; Wang, X.; Sun, T. T.; Dou, H. L.; Lin, X. M. Ophthalmology 2012, 119, 2290-2297.

6. Murray, I. J.; Makridaki, M.; van der Veen, R. L.; Carden, D.; Parry,

N. R.; Berendschot, T. T. Invest. Ophthalmol. Vis. Sci. 2013, 54, 1781-1788.

7. Huang, Y. M.; Dou, H. L.; Huang, F. F.; Xu, X. R.; Zou, Z. Y.; Lu,

X. R.; Lin, X. M. Br. J. Ophthalmol. 2015, 99, 371-375.

8. Thürmann, P. A.; Schalch, W.; Aebischer, J. C.; Tenter, U.; Cohn, W. Am. J. Clin. Nutr. 2005, 82, 88-97.

9. Schalch, W.; Cohn, W.; Barker, F. M.; Kõpcke, W.; Mellerio, J.; Bird, A. C.; Robson, A. G.; Fitzke, F. F.; van Kuijk, F. J. Arch. Biochem. Biophys. 2007, $458,128-135$.

10. Obana, A.; Tanito, M.; Gohto, Y.; Okazaki, S.; Gellermann, W.; Bernstein, P. S. PLoS One 2015, 10, e0139257.

11. Evans, M.; Beck, M.; Elliott, J.; Etheve, S.; Roberts, R.; Schalch, W. Eur. J. Nutr. 2013, 52,1381-1391.

12. Hartmann, D.; Thürmann, P. A.; Spitzer, V.; Schalch, W.; Manner, B.; Cohn, W. Am. J. Clin. Nutr. 2004, 79, 410-417.

13. Sato, Y.; Kobayashi, M.; Itagaki, S.; Hirano, T.; Noda, T.; Mizuno, S.; Sugawara, M.; Iseki, K. Biopharm. Drug Dispos. 2011, 32, 151-158.

14. Sowmya, P. R.; Arathi, B. P.; Vijay, K.; Baskaran, V.; Lakshminarayana, R. Chromatographia 2014, 77, 1633-1642.

15. Guidance for Industry: Bioanalytical Method Validation, U.S Department of Health and Human Service, Food and Drug Administration, Center for Drug Evaluation and Research (CDER), Centre for Veterinary Medicine (CVM), May 2001. 